\title{
High Density of Si Nanodots in Silicon Oxide Nanowires by Electron Beam Irradiation
}

\author{
Gyeong-Su Park,* Jun Ho Lee, ${ }^{*}$ Sung Heo, ${ }^{*}$ Hyuksang Kwon,** Juyeon Park,** and Seong Keun \\ $\operatorname{Kim} * *$ \\ * Analytical Engineering Group, Samsung Advanced Institute of Technology, PO Box 111, Suwon, \\ 440-600, South Korea \\ ** Department of Biophysics and Chemical Biology and Department of Chemistry, Seoul National \\ University, Seoul, 151-747, South Korea
}

The current semiconductor industry could be readily transformed into an immensely useful future technology if one could produce, on an industrial scale, Si nanodots of controllable sizes embedded in a $\mathrm{SiO}_{2}$ matrix. Methods of producing $\mathrm{Si}$ nanodots embedded in films of silicon oxide and silicon nitride abound, but fabrication of Si nanodots in a nanowire of these materials is very rare despite the fact that nanowire architecture enhances the charge collection and transport efficiencies for solar cells and field-effect transistors.

We synthesized the core-shell and gold-nanoparticle-embedded silicon oxide nanowires by simply heating, to $1273 \mathrm{~K}$ in a micro-chamber, an n-type (100) silicon wafer onto which a thin $(\sim 20 \mathrm{~nm})$ gold layer had been deposited. In this study we have fabricated a high-density array of size controlled silicon nanodots from the silicon oxide nanowires using electron-beam irradiation. The main instrument we used in this study for electron-beam irradiation and microscopy was our 300-kV monochromated and aberration corrected FEI TITAN field emission transmission electron microscope (FETEM) equipped with a high-resolution Gatan Tridieum 865ER imaging spectrometer.

Energy-filtered transmission electron microscopy (EF-TEM) gave the plasmon loss images of $\mathrm{Si}$ filtered at $17 \mathrm{eV}$ (Fig. 1, for two different diameters of nanowire) [1], which show that the number of $\mathrm{Si}$ nanodots formed increases with irradiation time. The second images of each figure show that $\mathrm{Si}$ atoms (red arrows) are initially aggregated around the $c$-SRO core and sheathe the $c$-SRO core with a thickness of about 1 to $1.3 \mathrm{~nm}$ (green ring). As the irradiation time becomes longer, more Si atoms aggregate into nanodots in the $a-\mathrm{SiO}_{2}$ matrix until eventually a high density of $\mathrm{Si}$ nanodots that are 2 $\sim 4 \mathrm{~nm}$ in size are formed in the $a-\mathrm{SiO}_{2}$ shell.

Electron transport properties of the $c-\mathrm{SRO} / a-\mathrm{SiO}_{2}$ core-shell nanowire before and after $\mathrm{Si}$ nanodot formation were investigated using a scanning tunneling microscope (STM) installed in a TEM holder. The TEM image of Fig. 2(a) shows that the $c$-SRO core was $7 \mathrm{~nm}$ in diameter and the distance between the probe and the electrode was $215 \mathrm{~nm}$. After contact was made between the Pt-Ir probe and the nanowire, we generated Si nanodots in the latter by using electron-beam irradiation at $25 \mathrm{~A} / \mathrm{cm}^{2}$ for $5 \mathrm{~min}$. Figure 2(b) gives the plasmon loss image for Si recorded after the Si nanodot formation, which clearly shows that a high number density of Si nanodots was successfully formed in the nanowire. When we apply a maximum bias voltage of $\pm 70 \mathrm{~V}$ to the original nanowire labeled A, we obtained the current-voltage (I-V) curve shown in Fig. 2(c). It has a broad bump in current that suggests electron accumulation in the core-shell nanowire due to high voltage stress. Figure 2(d) shows the difference in the $I-V$ curve of the nanowire before and after the Si nanodot formation. 
Despite the contact resistance between the probe and the nanowire, a maximum current of $15 \mathrm{nA}$ measured at $24 \mathrm{~V}$ across the nanowire (i.e., with a resistance of $\sim 1.6 \mathrm{G} \Omega$ ) that contains the $\mathrm{Si}$ nanodots is remarkable in comparison with the original nanowire that exhibits the characteristics of an insulator. The dramatic electrical transition from an insulator to a semiconductor may be due to the transport of electrons through the Si-rich phase surrounding the $c$-SRO core as well as the sequential electron tunneling through the $a$-Si nanodots. It is also to be noted that conduction displays a staircase-like behavior in the $I-V$ curve with a sudden change in current.[2] Monochromated EELS spectra and aberration-corrected STEM images of the silicon oxide nanowires after Si nanodot formation will be presented.

\section{References}

[1] A. Yurtsever et al., Appl. Phys. Lett. 89 (2006) 151920.

[2] G-S. Park et al., Nano Lett. 9 (2009) 1780.
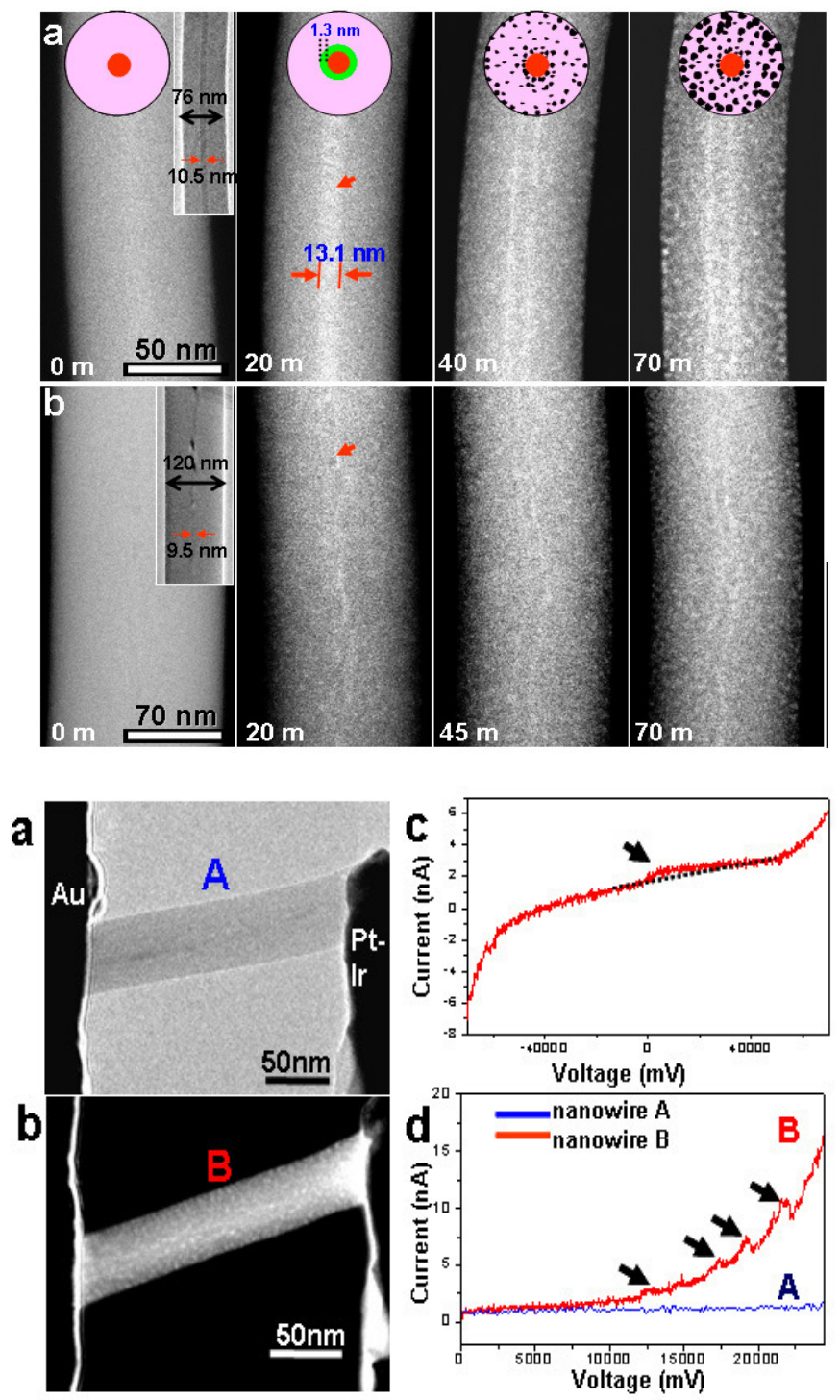

Fig. 1. Formation of Si nanodots in the $c$ $\mathrm{SRO} / a-\mathrm{SiO}_{2}$ core-shell nanowires during electron-beam irradiation at room temperature. The electron energy was 300 $\mathrm{keV}$ and the beam intensity was $1.2 \mathrm{~A} / \mathrm{cm}^{2}$. $(\mathbf{a}, \mathbf{b})$ Irradiation-time-dependent plasmon loss images of a 76-nm-diameter core-shell nanowire with a $c$-SRO core diameter of 10.5 $\mathrm{nm}$ and a 120-nm-diameter nanowire with a core diameter of $9.5 \mathrm{~nm}$. The circular diagrams in (a) schematically depict a cross-sectional view of the core-shell nanowire during irradiation, which represents the intrinsic $c$ SRO core (red), the Si-sheath around the core (green), the $\mathrm{Si}$ nanodots (black), and the $a$ $\mathrm{SiO}_{2}$ shell (pink).

Fig. 2. Electron charging and current conduction through the core-shell nanowire. (a) TEM image of a 61-nm-diameter core-shell nanowire in contact with an STM tip (Pt-Ir) inside TEM. (b) Si plasmon loss image of the nanowire in (a) after $5 \mathrm{~min}$ of electron-beam irradiation at $25 \mathrm{~A} / \mathrm{cm}^{2}$. The nanowires before and after electron-beam irradiation are each labeled A and $\mathrm{B}$, respectively. (c) $I-V$ curve for nanowire A (with no Si nanodots formed within) over the voltage range of $-70 \mathrm{~V}$ to $+70 \mathrm{~V}$. (d) Current vs. voltage $(I-V)$ curves for the two nanowires $\mathrm{A}$ and $\mathrm{B}$ with a bias voltage of up to $25 \mathrm{~V}$. 